\title{
PROPRIEDADES FÍSICO-QUÍMICAS EM POLPAS DE FRUTOS DO CERRADO IN NATURA E LIOFILIZADA.
}

Physicochemical properties in pulps of fruits of the cerrado in the wild and lyophilized.

Propiedades fisicoquímicas en pulpas de frutos del cerrado en la naturaleza y liofilizados

\section{André Leonardo dos Santos ${ }^{*}{ }^{1,2}$, Camila Mariane da Silva Soares ${ }^{2}$, Patrícia Oliveira Vellano $^{2}$, Rômulo Alves Morais ${ }^{1,2}$, Valéria Gomes Momenté ${ }^{3}$, Glêndara Aparecida de} Souza Martins ${ }^{1,2}$, Adriana Régia Marques de Souza ${ }^{2}$

${ }^{1}$ Laboratório de Cinética e Modelagem de Processos (LaCiMP) - Universidade Federal do Tocantins

${ }^{2}$ Programa de Pós-Graduação em Ciência e Tecnologia de Alimentos (PPGCTA -UFT) - Universidade Federal do Tocantins

${ }^{3}$ Docente do Programa de Graduação em Engenharia de alimentos (PGEA -UFG) - Universidade Federal do Tocantins.

*Correspondência: Laboratório de Cinética e Modelagem de Processos, Escola de Engenharia de Alimentos, Universidade Federal do Tocantins, Quadra 109 Norte, Avenida NS 15, ALCNO-14, Plano Diretor Norte, Palmas - TO, CEP: 77001-090.e-mail: anddreleo@gmail.com

Artigo recebido em 10/03/2020 aprovado em 27/03/2020 publicado em 31/03/2020.

\section{RESUMO}

Com 204,7 milhões de hectares o cerrado é a segunda maior extensão vegetal brasileira. Os frutos do cerrado possuem sabor e aroma característicos e podem se apresentar de formas variadas além de serem boas fontes de nutrientes. A conservação por liofilização é utilizada para preservar características nutritivas e compostos bioativos. Este trabalho objetivou caracterizar a polpa de buriti, murici, cajá e tamarindo in natura quanto a suas propriedades físico-químicas e valor nutricional, além de compará-los com pó obtido através da liofilização. Para a obtenção do pó, foi adicionado ás polpas 19,\% do encapsulante AmidoMax2500, as polpas foram congeladas a $-70^{\circ} \mathrm{C}$ por 24 horas e em seguidas foram submetidas ao processo de liofilização por 72 horas. A liofilização foi eficaz na concentração de alguns componentes tais como $\mathrm{pH}$, acidez, sólidos solúveis, cinzas e lipídeos. Os teores de ácido ascórbico foram mantidos e em alguns casos houve uma maior concentração. Mostrou-se eficiente ainda na preservação da cor das frutas e na retirada de água livre. O uso da liofilização é eficaz na concentração e preservação dos compostos nutritivos e bioativos presente nos frutos.

Palavras-chave: Secagem, liofilização, caracterização

\section{ABSTRACT}

With 204.7 million hectares the cerrado is the second largest extension of Brazilian vegetation. The fruits of the cerrado have a characteristic flavor and aroma and can be presented in various forms and are good sources of nutrients. Freeze-drying preservation is used to preserve nutritional characteristics and bioactive compounds. This work aimed to characterize the pulp of buriti, murici, cajá and tamarindo in natura as to their physicochemical properties and nutritional value, and to compare them with powder obtained through lyophilization. To obtain the powder, 19\% of the AmidoMax2500 encapsulant was added to the pulps, the pulps were frozen at $-70{ }^{\circ} \mathrm{C}$ for 24 hours and then subjected to the lyophilization process for 72 hours. Lyophilization was effective in concentrating some components such as $\mathrm{pH}$, acidity, soluble solids, ashes and lipids. Ascorbic acid levels were maintained and in some cases there was a higher concentration. It was also efficient in preserving the color of the fruits and removing free water. The use of lyophilization is effective in the concentration and preservation of nutritive and bioactive compounds present in the fruit pulp.

Keywords:: Drying, freeze drying, characterization. 


\section{RESUMEN}

Con 204,7 millones de hectáreas, el cerrado es la segunda extensión más grande de vegetación brasileña. Los frutos del cerrado tienen un sabor y aroma característicos y pueden presentarse en varias formas y son buenas fuentes de nutrientes. La conservación por liofilización se utiliza para preservar las características nutricionales y los compuestos bioactivos. Este trabajo tuvo como objetivo caracterizar la pulpa de buriti, murici, cajá y tamarindo in natura en cuanto a sus propiedades fisicoquímicas y valor nutricional, y compararlos con el polvo obtenido mediante liofilización. Para obtener el polvo, se añadió el 19\% del encapsulante AmidoMax2500 a las pulpas, las pulpas se congelaron a $-70{ }^{\circ} \mathrm{C}$ durante 24 horas y luego se sometieron al proceso de liofilización durante 72 horas. La liofilización fue efectiva para concentrar algunos componentes como el pH, la acidez, los sólidos solubles, las cenizas y los lípidos. Los niveles de ácido ascórbico se mantuvieron y en algunos casos hubo una concentración más alta. También fue eficiente para preservar el color de las frutas y eliminar el agua libre. El uso de liofilización es efectivo en la concentración y preservación de compuestos nutritivos y bioactivos presentes en la pulpa de fruta.

Descriptores:: Secado, liofilización, caracterización.

\section{INTRODUÇÃO}

Cerrado é o segundo maior bioma brasileiro, ocupando uma área de 2.036.448 $\mathrm{km}^{2}$, aproximadamente $22 \%$ do território nacional. Sua área contínua está presente nos estados de Goiás, Tocantins, Mato Grosso, Mato Grosso do Sul, Minas Gerais, Bahia, Maranhão, Piauí, Rondônia, Paraná, São Paulo e Distrito Federal, além de trechos nos estados do Amapá, Roraima e Amazonas (SILVA et al.,2017).

Os frutos nativos do cerrado possuem sabor e aroma característicos e podem se apresentar de formas variadas além de ser uma boa fonte de nutrientes (DA PAZ, 2014). Dentre esses frutos podemos citar o buriti (Mauritia flexuosa), cajá (Spondias mombin), murici (Byrsonima verbascifolia) e o tamarindo (Tamarindus indica), estes são principalmente consumidos frescos e servem como matéria prima para diversos produtos tais como, licores, geleias, sorvetes, picolés, doces, sucos e néctares.

Visando a conservação das frutas, vários métodos de secagem são utilizados, dentre estes, a liofilização que é utilizada para preservar frutas bem como suas características nutritivas e compostos bioativos. Por utilizar baixar temperatura durante todo o processo, a liofilização não degrada os compostos voláteis, preservando assim o aroma e sabor característico das frutas (VIEIRA et al., 2012).

Por provocar alterações mínimas nas características sensoriais e nos valores nutricionais, a liofilização aumenta a estabilidade do produto, podendo este ser armazenado em temperatura ambiente, e ainda reduz o peso e o volume do produto final, estes benefícios possibilitam a redução nos custos com embalagem, armazenamento e transporte (BEZERRA, 2014).

Diante da escassez de informações sobre o processo de liofilização em polpa de frutos do cerrado e seu possível potencial de inserção no mercado, o presente trabalho objetivou caracterizar os frutos de buriti, murici, cajá e tamarindo in natura quanto a suas propriedades físico-químicas e valor nutricional, além de compará-los com pó obtido através da liofilização.

\section{MATERIAIS E MÉTODOS}

As polpas de frutas congeladas foram adquiridas na feira livre de Palmas-TO. Foram escolhidas polpas de frutos provenientes do cerrado: Murici, Tamarindo, Cajá e Buriti. Todos os procedimentos e experimentos foram realizados nos Laboratórios da Fundação Universidade Federal do Tocantins, campus de Palmas. O experimento foi conduzido no período de agosto a novembro de 2015 e todas as análises foram feitas em triplicata. 
As polpas foram descongeladas e em função do alto teor de umidade das polpas foi adicionado 19,1\% do encapsulante AmidoMax2500, para utilização desta percentagem foram realizados testes prévios, o encapsulante foi utilizado por ter ação antiumectante auxiliando na desidratação, em seguida as amostras foram homogeneizadas em Agitador Mecânico Eletrônico (Macro -Q250) durante cinco minutos.

As polpas homogeneizadas foram colocadas em potes plásticos com capacidade de $50 \mathrm{ml}$ e submetidas a congelamento em ar estático utilizando ultra freezer (marca Thermoscientific modelo 88000), a temperatura utilizada foi $-70^{\circ} \mathrm{C}$ por 24 horas. As polpas congeladas foram conduzidas a um liofilizador (marca Liotop modelo L101), que consiste em uma câmara de vácuo, condensador, unidade de refrigeração e bomba de vácuo. O tempo médio para a liofilização das amostras foi de 72h. Em seguida, os produtos provenientes da liofilização foram triturados e, logo após, foram acondicionados em frascos fechados, protegidos da luz e armazenados a temperatura ambiente.

As análises de umidade, acidez titulavél total, cinzas, $\mathrm{pH}$, lipídeos, sólidos solúveis totais, e vitamina $\mathrm{C}$ foram realizadas de acordo com a metodologia descrita por (IAL, 2008). A análise instrumental de cor foi determinada usando um colorímetro digital (Minolta CR400, fonte de luz D65 em espaço de cor $L^{*}, a^{*}$ e b* do sistema CIE L*a*b). O aparelho foi calibrado com placa branca padrão, seguindo as instruções do fabricante. Os resultados foram expressos em $\mathrm{L}^{*}$ (luminosidade) que varia de 0 (preto) a 100 (branco); o a* varia de -a* (verde;) a $+a^{*}$ (vermelho) e o b* de $-b^{*}$ (azul) $a+b^{*}$ (amarelo) (MCGUIRE, 1992).

Os efeitos dos tratamentos foram comparados pela análise de variância e quando houve significância foi utilizado o Teste de Tukey a 5\% $(\mathrm{p}<0,05)$ para identificar as diferenças. As análises de variância e teste de médias foram realizadas segundo as técnicas usuais do software SISVAR (FERREIRA, 2011).

\section{RESULTADOS E DISCUSSÃO}

Os resultados das análises físico-químicas das polpas de buriti, cajá, murici e tamarindo in natura e liofilizada encontram-se na Tabela 1.

Quantos aos valores de $\mathrm{pH}$, não houve diferença estatística para polpa buriti in natura e liofilizada, ambas apresentaram um valor de 3,76. As demais polpas apresentaram diferença significativa com valores de $\mathrm{pH}$ variando entre 2,73 para polpa liofilizada de tamarindo e 3,70 para polpa in natura de muricí. Essa tendência à redução do $\mathrm{pH}$ em amostras liofilizada foi reportada em um estudo conduzido por Ibiapina et al., (2018) em polpa de fruta do tipo detox, onde seus resultados foram, 3,15 para polpa in natura e 3,12 para polpa liofilizada. Essa redução do pH ocorre pela redução do conteúdo de água nas polpas liofilizadas provocando assim uma concentração dos compostos no produto liofilizado (LAMEIRO et al. 2019).

Os teores de acidez titulável total encontrados neste estudo apresentaram um aumento significativo para polpas liofilizadas, destacando-se as polpas de cajá (24,3) e tamarindo (22,02). Resultados semelhantes foram encontrados por Oliveira et al., (2011) ao caracterizarem físico-quimicamente a polpa de sapoti, obtendo resultados de acidez de $0,083 \%$ e $0,247 \%$ para polpa in natura e liofilizada, respectivamente. Vieira et al., (2015) em estudos semelhantes com polpa de acerola também relatou um aumento significativo no teor de acidez, a polpa in natura apresentou $1,44 \%$ enquanto a liofilizada apresentou 3,03\%. De forma semelhante aos resultados obtidos para $\mathrm{pH}$, o aumento da acidez está relacionado com a perda de água na amostra e a 
concentração dos compostos provocada pelo processo

\begin{tabular}{|c|c|c|c|c|c|c|c|c|}
\hline \multirow{3}{*}{ Análises } & \multicolumn{8}{|c|}{ Amostras } \\
\hline & \multicolumn{2}{|c|}{ Buriti } & \multicolumn{2}{|c|}{ Cajá } & \multicolumn{2}{|c|}{ Murici } & \multicolumn{2}{|c|}{ Tamarindo } \\
\hline & In natura & Liofilizada & In natura & Liofilizada & In natura & Liofilizada & In natura & $\underline{\text { Liofilizada }}$ \\
\hline $\mathrm{pH}$ & $3,76 \pm 0,0^{\mathrm{a}}$ & $3,76 \pm 0,0^{\mathrm{a}}$ & $3,56 \pm 0,2^{\mathrm{a}}$ & $2,93 \pm 0,0^{\mathrm{b}}$ & $3,70 \pm 0,0^{\mathrm{a}}$ & $3,46 \pm 0,0^{\mathrm{b}}$ & $3,28 \pm 0,0^{\mathrm{a}}$ & $2,73 \pm 0,0^{\mathrm{b}}$ \\
\hline $\mathrm{ATT}^{1}$ & $1,97 \pm 0,0^{b}$ & $4,22 \pm 0,0^{\mathrm{a}}$ & $2,49 \pm 0,0^{b}$ & $24,33 \pm 0,1^{\mathrm{a}}$ & $2,83 \pm 0,1^{b}$ & $17,85 \pm 0,6^{\mathrm{a}}$ & $7,29 \pm 0,0^{\mathrm{b}}$ & $22,02 \pm 2,8^{\mathrm{a}}$ \\
\hline $\mathrm{SST}^{2}$ & $7,75 \pm 0,0^{\mathrm{a}}$ & $8,08 \pm 0,0^{b}$ & $6,07 \pm 0,0^{\mathrm{b}}$ & $13,75 \pm 0,0^{\mathrm{a}}$ & $5,25 \pm 0,0^{\mathrm{b}}$ & $6,37 \pm 0,2^{\mathrm{a}}$ & $21,00 \pm 0,0^{\mathrm{a}}$ & $21,00 \pm 0,0^{\mathrm{a}}$ \\
\hline Umidade & $64,38 \pm 0,7^{\mathrm{a}}$ & $3,29 \pm 1,2^{b}$ & $93,72 \pm 0,0^{\mathrm{b}}$ & $13,03 \pm 1,7^{\mathrm{a}}$ & $88,95 \pm 1,0^{\mathrm{b}}$ & $8,66 \pm 0,2^{\mathrm{a}}$ & $75,80 \pm 0,3^{\mathrm{a}}$ & $13,69 \pm 0,2^{b}$ \\
\hline Cinzas & $0,78 \pm 0,1^{\mathrm{b}}$ & $2,25 \pm 0,0^{\mathrm{a}}$ & $0,31 \pm 0,0^{\mathrm{b}}$ & $3,88 \pm 0,0^{\mathrm{a}}$ & $0,29 \pm 0,0^{\mathrm{b}}$ & $2,47 \pm 0,0^{\mathrm{a}}$ & $0,77 \pm 0,0^{\mathrm{b}}$ & $3,28 \pm 0,2^{\mathrm{a}}$ \\
\hline Lipídeos & $30,74 \pm 0,4^{\mathrm{a}}$ & $38,66 \pm 1,0^{\mathrm{b}}$ & $1,42 \pm 0,9^{\mathrm{a}}$ & $0,41 \pm 0,1^{\mathrm{a}}$ & $13,98 \pm 0,3^{\mathrm{a}}$ & $9,57 \pm 0,7^{b}$ & $0,30 \pm 0,0^{\mathrm{b}}$ & $0,93 \pm 0,0^{\mathrm{a}}$ \\
\hline
\end{tabular}

de liofilização (LAMEIRO et al. 2019).

Tabela 1 - Valores de pH, acidez titulável total (ATT), sólidos solúveis totais (SST), umidade, cinzas e lipídeos em polpas de frutos do cerrado in natura e liofilizada.

${ }^{1}$ expresso em mg de ácido cítrico/100g de polpa; ${ }^{2}$ expreso em ${ }^{\circ}$ Brix; Dados expressos com média de triplicata \pm esvio padrão \pm desvio padrão. As médias seguidas com a mesma letra na horizontal não diferem estatisticamente entre si, pelo teste de Tukey, a nível de $5 \%$ de probabilidade.

Quanto aos valores de sólidos solúveis totais, não houve diferença significativa na polpa de tamarindo (21 ${ }^{\circ}$ Brix $)$, tanto para polpa in natura quando para polpa liofilizada. As demais polpas de buriti, cajá e muricí apresentaram diferença significativa entre os tratamentos. A polpa cajá liofilizada foi a que apresentou maior aumento no teor de sólidos solúveis $\left(13,75^{\circ}\right.$ Brix $)$ e o menor teor foi encontrado foi na polpa in natura de murici $(5,25$ ${ }^{\circ}$ Brix). Comportamento semelhante foi relatado nos estudos de Duarte et al., (2018) com lichia (Lichi chinensis) liofilizada com diferentes concentrações de maltodextrina, onde a polpa in natura apresentou um valor de $15,66^{\circ}$ Brix e polpa liofilizada apresentou valores de $82,49^{\circ}$ Brix.

Os teores de umidade variaram entre 64 e $93 \%$ para as polpas in natura e de 3 a $13 \%$ para as polpas liofilizada. Resultados semelhantes foram descritos por Oliveira et al., (2010) em seus estudos com polpa de ubaia onde a fruta in natura foi aferida com $92,47 \%$ de umidade e a polpa liofilizada foi aferida com $14,25 \%$. Essa redução no teor de umidade pode ser explicada pelo fato da liofilização ser um processo que elimina água com eficiência devido ao uso do vácuo e baixas temperaturas (CHITARRA, 2005).
Quanto ao teor de cinzas, é possível observar uma diferença significativa entre as polpas in natura e as liofilizadas. O processo que liofilização provocou um aumento no teor de cinzas, o resultado mais expressivo foi observado na polpa de cajá. Bezerra (2014) em seus estudos sobre polpa de marolo liofilizada também observou um aumento de $(1,42 \%$ in natura) para (3,32\% liofilizada) no teor de cinzas.

Os resultados expressos para os teores de lipídeos mostram que não houve diferença significativa entre os tratamentos apresentados para as polpas de cajá e tamarindo, no entanto houve uma redução no teor de lipídeos na polpa de murici liofilizada $(9,57 \%)$ quando comparada com a polpa in natura $(13,98 \%)$. Apesar da redução no teor de lipídeos, o muricí fruto que apresenta $72,61 \%$ de ácidos graxos insaturados dentre eles ácido linolêioco $(\omega-3)$ e oleico ( $\omega-6)$ sendo esses essenciais para a saúde humana (HOLANDA, 2016). Na polpa de burití observa-se uma concentração significativa no teor de lipídeos, de $(30,74 \%)$ na polpa in natura para $(38,66 \%)$ na polpa liofilizada, esse mesmo comportamentos foi descrito por Faria et al., (2016) onde descreveu concentração de lipídeos em casca de jaboticaba com o processo de liofilização, onde a casca 
in natura apresentou $0,67 \%$ em quanto a casa

liofilização apresentou $1,86 \%$.
A Tabela 2 expressa os valores de ácido ascórbico para as amostras de buriti, cajá, murici e tamarindo.

Tabela 2 - Valores de ácido ascórbico (AA) em polpas de frutos do cerrado in natura e liofilizadas

\begin{tabular}{|c|c|c|c|c|c|c|c|c|}
\hline \multirow{3}{*}{ Análise } & \multicolumn{8}{|c|}{ Amostras } \\
\hline & \multicolumn{2}{|c|}{ Buriti } & \multicolumn{2}{|c|}{ Cajá } & \multicolumn{2}{|c|}{ Murici } & \multicolumn{2}{|c|}{ Tamarindo } \\
\hline & In natura & Liofilizada & In natura & Liofilizada & In natura & Liofilizada & In natura & Liofilizada \\
\hline AA & $1,04 \pm 0,0^{\mathrm{a}}$ & $0,96 \pm 0,0^{\mathrm{a}}$ & $0,72 \pm 0,2^{\mathrm{a}}$ & $0,79 \pm 0,0^{\mathrm{b}}$ & $1,22 \pm 0,0^{\mathrm{a}}$ & $0,52 \pm 0,4^{b}$ & $0,46 \pm 0,9^{a}$ & $0,26 \pm 0,0^{b}$ \\
\hline
\end{tabular}

Dados expressos com média de triplicata \pm desvio padrão. As médias seguidas com a mesma letra na horizontal não diferem estatisticamente entre si, pelo teste de Tukey, a nível de $5 \%$ de probabilidade.

Com relação aos resultados do teor de ácido ascórbico, não houve diferença significativa para a polpa de buriti. É possível observar que após processo de liofilização houve uma redução no teor AA nas amostras de murici e tamarindo $(0,52$ e 0,26, respectivamente). Esses resultados corroboram como os estudos de Bezerra, (2014) onde autor relatou a degradação da vitamina $\mathrm{C}$ após o processo de liofilização em seu estudo com marolo, onde fruto in natura apresentava 81,50 (mg/100g) de vitamina C, enquanto o fruto liofilizado apresentou 63,07 $(\mathrm{mg} / 100 \mathrm{~g})$. Essas perdas podem estar relacionadas à alta porosidade promovida pelo processo de liofilização, aumentando a superfície de contato do produto com o oxigênio, e à formação de cristais de gelo durante o congelamento, resultando em grande destruição do tecido celular e, consequentemente, maior exposição do nutriente ao oxigênio (ALBERTON, 2014). A polpa de cajá, ao contrário das demais polpas estudadas, apresentou uma concentração de AA após o processo de liofilização, a polpa in natura apresentou um valor de $(0,72)$ enquanto a polpa liofilizada apresentou $(0,79)$, corroborando com os estudos de Vieira et al., (2015), em seus relatos a polpa de acerola in natura apresentou teor de vitamina C de 416,14(mg/100g) e após a liofilização o teor de vitamina $\mathrm{C}$ foi elevado para $1.571,05(\mathrm{mg} / 100 \mathrm{~g})$.

A tabela 3 apresenta os valores obtidos para os parâmetros de cor das amostras de buriti, cajá, murici e tamarindo ( $\left.\mathrm{L}^{*}, \mathrm{a}^{*} \mathrm{e} \mathrm{b}\right)$.

Em todas as polpas estudadas observou-se a predominância da cor amarela $\left(b^{*}\right)$ sobre vermelha $\left(\mathrm{a}^{*}\right)$, isso pode ser constatado pelos valores elevados de $\left(b^{*}\right)$, em destaque para polpas de cajá e murici, as polpas destes frutos tanto in natura quando liofilizadas foram as que apresentaram os valores mais elevados de $\left(b^{*}\right)$. As amostras de buriti, cajá, e murici apresentaram uma redução dos valores de (a*) após o processo de liofilização, os valores obtidos mostram que as polpas in natura apresentam cor mais avermelhada que a polpa liofilizada

Tabela 3 - Valores de L* a* e b* em polpas de frutos do cerrado in natura e liofilizada.

\begin{tabular}{|c|c|c|c|c|c|c|c|c|}
\hline \multirow{3}{*}{ Cor } & \multicolumn{8}{|c|}{ Amostras } \\
\hline & \multicolumn{2}{|c|}{ Buriti } & \multicolumn{2}{|c|}{ Cajá } & \multicolumn{2}{|c|}{ Murici } & \multicolumn{2}{|c|}{ Tamarindo } \\
\hline & In natura & Liofilizada & In natura & Liofilizada & In natura & Liofilizada & In natura & Liofilizada \\
\hline $\mathrm{L}^{*}$ & $17,16 \pm 0,0^{b}$ & $20,75 \pm 0,0^{\mathrm{a}}$ & $46,35 \pm 0,0^{\mathrm{a}}$ & $19,99 \pm 0,0^{\mathrm{b}}$ & $45,81 \pm 0,0^{\mathrm{b}}$ & $19,10 \pm 0,0^{\mathrm{a}}$ & $11,71 \pm 0,0^{\mathrm{b}}$ & $13,13 \pm 0,0^{\mathrm{a}}$ \\
\hline$a^{*}$ & $8,55 \pm 0,0^{\mathrm{a}}$ & $6,93 \pm 0,0^{b}$ & $10,56 \pm 0,0^{\mathrm{a}}$ & $6,85 \pm 0,1^{b}$ & $8,76 \pm 0,0^{b}$ & $5,73 \pm 0,0^{\mathrm{a}}$ & $5,28 \pm 0,0^{b}$ & $6,90 \pm 0,0^{\mathrm{a}}$ \\
\hline
\end{tabular}


As médias seguidas com a mesma letra na horizontal não diferem estatisticamente entre si, pelo teste de Tukey, a nível de 5\% de probabilidade

Dentre as polpas in natura a tonalidade mais escura foi encontrada em tamarindo $\left(\mathrm{L}^{*}=11,71\right)$ e a mais clara foi encontrada em cajá $\left(L^{*}=46,35\right)$, após a liofilização o buriti foi a polpa mais clara $\left(\mathrm{L}^{*}=20,75\right)$ e a mais escura foi a de tamarindo $\left(L^{*}=13,13\right)$. A liofilização provocou um aumento da luminosidade nas polpas de buriti e tamarindo (20,75 e 13,13, respectivamente). Lunardi et al., (2015) estudaram parâmetros de cor em abacaxi liofilizado relatando um aumento dos valores de luminosidade, o valor encontrado para abacaxi in natura foi de $\left(\mathrm{L}^{*}=66,2\right)$ enquanto o fruto liofilizado apresentou $\left(\mathrm{L}^{*}=76\right)$. $\mathrm{O}$ aumento da luminosidade nas polpas liofilizadas de buriti e tamarindo demonstra o clareamento das amostras após o processo de liofilização. Esse clareamento por esta associado à adição do encapsulante de cor branca inserido nas polpas antes do processo de liofilização.

\section{CONCLUSÃO}

$\mathrm{O}$ processo de liofilização se mostrou adequado para a concentração de alguns componentes tais como $\mathrm{pH}$, acidez, sólidos solúveis, cinzas e lipídeos. Os teores de ácido ascórbico foram mantidos e em alguns casos houve concentração. A liofilização se mostrou eficiente ainda na preservação da cor das frutas e na retirada de água livre. O pó obtido após o processo de liofilização é mais estável, leve e menos volumoso que a polpa in natura podendo reduzir o custo com embalagem, transporte e armazenamento.

\section{AGRADECIMENTOS}

A equipe do Laboratório de Cinética e Modelagem de Processos, e ao CNPq pelo apoio financeiro para o desenvolvimento dessa pesquisa.
Todos os autores declararam não haver qualquer potencial conflito de interesses referente a este artigo.

\section{REFERÊNCIAS}

ALBERTON, ANDRESSA CAROLINE MAZZUCHELLO. Caracterização da manga Tommy Atkins in natura e após a liofilização. Trabalho de conclusão de curso (Graduação em Engenharia de alimentos). Universidade Tecnológica Federal do Paraná - UTFPR. Medianeira, 2014

BEZERRA, T. S. Caracterização física, química e morfológica de polpa de marolo liofilizada. Tese (Pós-Graduação em Ciência dos Alimentos). Universidade Federal de Lavras. Lavras, MG. 141 p., 2014.

CANUTO, Gisele André Baptista et al. Caracterização físico-química de polpas de frutos da Amazônia e sua correlação com a atividade anti-radical livre. Revista Brasileira de Fruticultura, 2010.

DA PAZ, Josiane Gonçalves et al. Análise da composição nutricional e de parâmetros físicoquímicos do pequi (Caryocar brasiliense camb) in natura. Revista Científica Linkania Master, v. 1, n. 8, 2014.

DE CASTRO, D. S. et al. Caracterização física e físico-química de polpa de buriti (Mauritia flexuosa). Revista Verde de Agroecologia e Desenvolvimento Sustentável, v. 9, n. 2, p. 117-120, 2014.

DUARTE, M. T. L. et al. Qualidade da polpa de lichia em pó, obtida pelo processo de liofilização. Tese Universidade Federal de Campina Grande, Centro de Ciência e Tecnologia, 2018.

FARIA, G.S. et al. Caracterização Química da casca de Jabuticaba (Myrciaria jabuticaba) liofilizada e sua aplicação em leite fermentado potencialmente simbiótico. JORNAL DE CIÊNCIAS BIOMÉDICAS E SAÚDE, v. 2, n. 1, p. 2, 2016.

FERREIRA, D F. Sisvar: a Guide for its Bootstrap procedures in multiple comparisons. Ciência Agrotécnica, v.38, n.2, 2014. 
HOLANDA, L. C., Dissertação (Pós-Graduação em Química). Caracterização química e atividades biológicas do óleo vegetal da semente da espécie Byrsonima verbascifolia (L.) DC. Universidade Federal de Roraima 2016.

IAL (INSTITUTO ADOLFO LUTZ). Métodos físicoquímicos para análise de alimentos. 4 ed. São Paulo: IAL, 2008. 1018p.

IBIAPINA, A. et al. OBTENÇÃO DE PÓ DE POLPA DETOX UTILIZANDO LIOFILIZAÇÃO E SPRAY DRYING COMO MÉTODOS DE SECAGEM. GLOBAL SCIENCE AND TECHNOLOGY, v. 11, n. 3, 2018.

LAMEIRO, M. G. S et al. CARACTERÍSTICAS FÍSICO-QUÍMICAS DA AMORA-PRETA (Rubusfruticosus) E MIRTILO (Vacciniumasheireade) EM SEUS PRODUTOS LIOFILIZADOS. GLOBAL SCIENCE AND TECHNOLOGY, v. 12, n. 1, 2019.

LUNARDI, H; ROSA, G. S. Estudo da influência da liofilização nos parâmetros de cor de frutas tropicais. Blucher Chemical Engineering Proceedings, v. 2, n. 1, p. 1858-1863, 2015.

MCGUIRE. R.G. Reporting of objetive color measurements. HortScience, Alexandria, v.27, n.12, p. 1254-1255, Dec. 1992.

MONTEIRO, D.B; SOUSA, W. C.; PIRES, C. R. F.; 2, AZEVEDO, L. A.; BORGES, J.S.; CARACTERIZAÇÃO FÍSICO-QUÍMICA DO FRUTO E DA GELEIA DE MURICI (Brysonima crassifólia) ENCICLOPÉDIA BIOSFERA, Centro Científico Conhecer - Goiânia, v.11 n.21; p. 2015.

OLIVEIRA E. N.A et al. Obtenção de ubaia desidratada pelo processo de liofilização. Revista Brasileira de Tecnologia, v. 4, n. 02, p. 235-242, 2010.
OLIVEIRA, VALÉRIA SANTOS; AFONSO, MARCOS RODRIGUES AMORIM; COSTA, JOSÉ MARIA CORREIA. Caracterização físico-química e comportamento higroscópico de sapoti liofilizado. Revista Ciência Agronômica, v. 42, n. 2, p. $342-348,201$

SILVA, E. P. D., et al.. Characterization of chemical and mineral composition of marolo (Annona crassiflora Mart) during physiological development. Food Science and Technology, v. 37, n. 1, p. 13-18, 2017.

VIEIRA, A. P.; NICOLETI, J. F.; TELIS, V. R. N. Liofilização de fatias de abacaxi: avaliação da cinética de secagem e da qualidade do produto. Brazilian Journal of Food Technology, v.15, n.1, p.50-58, 2012

VIEIRA, A.C.A et al. Caracterização físico-química de polpa de acerola in natura e liofilizada para preparação de sorvetes. Blucher Chemical Engineering Proceedings, v. 1, n. 2, p. 3542-3546, 2015.

VIEIRA, R. F. Frutas nativas da região CentroOeste do Brasil. Embrapa Informação Tecnológica: Embrapa Recursos Genéticos e Biotecnologia, 2011. 